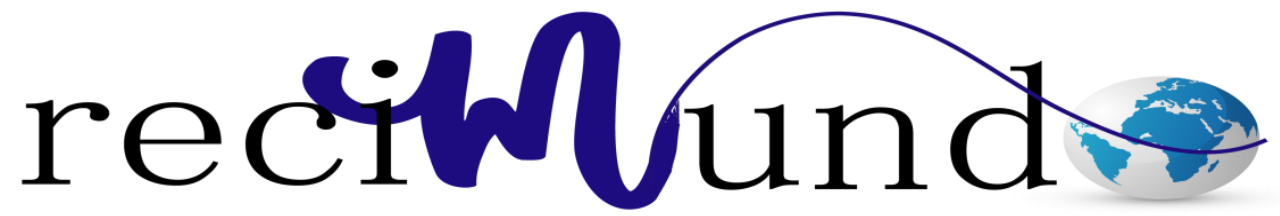

Revista Científica Mundo de la Investigación y el Conocimiento

Luis Alberto Anchundia Santana ${ }^{a}$; Robin Alejandro Torres ${ }^{\text {b }}$; Gilberto Giovanni Francisco Carranza Pacheco ${ }^{\mathrm{c}}$

Plan de acción para la gestión del riesgo ergonómico en el área de pelado, corte y desvenado a los que están expuestos los trabajadores en una empacadora de camarón

Revista Científica Mundo de la Investigación y el Conocimiento. Vol. 2 núm., 1, febrero, ISSN: 2588-073X, 2018, pp. 595-610

DOI: $10.26820 /$ recimundo/2.1.2018.595-610

Editorial Saberes del Conocimiento

Recibido: 05/12/2017

Aceptado: 10/02/2018 


\section{Plan de acción para la gestión del riesgo ergonómico en el área de pelado, corte y desvenado a los que están expuestos los trabajadores en una empacadora de camarón}

Vol. 2, núm. 1., (2018)

Luis Alberto Anchundia Santana; Robin Alejandro Torres; Gilberto Giovanni Francisco Carranza Pacheco

\section{RESUMEN}

El presente trabajo de investigación tiene el objetivo de identificar y evaluar los riesgos ergonómicos relacionados con la exposición a posturas forzadas y movimientos repetitivos en el área de pelado corte y desvenado de Empacadora así mismo elaborar y proponer un plan de acción para la gestión del riesgo ergonómico en esta área. La Empacadora se encuentra ubicada en la ciudad de Montecristi- Manabí, la población de estudio serán 126 mujeres que representa el $28 \%$ de la población total de la empresa, según reporte del servicio médico de la empresa es común recibir casos de trabajadores que aquejan molestias de tipo muscular en esta área, sea estas a nivel de miembros superiores, inferiores o espalda. Las actividades analizadas, son consideradas tareas con alta probabilidad en incurrir a alteraciones osteomusculares, dado la prevalencia en la adopción de posturas forzadas de pie y movimientos repetitivos. Se evalúa los riesgos generales del área a través de métodos reconocidos tales como el método de evaluación General de Riesgos de Instituto Nacional de Seguridad e Higiene de España (INSHT), se aplica métodos de evaluación ergonómica, el método CHECK LIST OCRA para movimientos repetitivos y OWAS para posturas forzadas de pie, para este análisis aplicamos percentiles 5 y 95, se aplica técnicas de encuestas a los trabajadores y entrevistas al personal de supervisión y dirección, una vez evaluados los riesgos ergonómicos en el área se pretende la elaboración de herramientas encaminadas a la gestión del riesgo ergonómico, tales como ejercicios de relajación, diseño de puesto de trabajo y plan de vigilancia de la salud, todas estas medidas serán enfocadas a la disminución de ausentismo laboral dada por la aparición de enfermedades de tipo osteomusculares.

Palabras clave: Ergonomía, riesgo del trabajo, enfermedad profesional, lesiones osteomusculares, pelado corte, desvenado. 


\section{Plan de acción para la gestión del riesgo ergonómico en el área de pelado, corte y desvenado a los que están expuestos los trabajadores en una empacadora de camarón}

Vol. 2, núm. 1., (2018)

Luis Alberto Anchundia Santana; Robin Alejandro Torres; Gilberto Giovanni Francisco

Carranza Pacheco

\section{ABSTRACT}

This research aims to identify and evaluate the ergonomic risks related to exposure to awkward postures and repetitive movements in the area of cutting peeled and deveined at the pocking likewise elaborate and propose an action plan for ergonomic risk management in this area. Packing is located in the city of Manabí-Montecristi, the study population will be of 126 women representing $28 \%$ of the total population of the company, according to a report of the medical service of the company is common to receive cases of workers with muscle pains, whether these are higher or lowen memebers or back. The activities analyzed, are considered high probability tasks incur in musculoskeletal disorders, given the prevalence in adopting awkward postures and repetitive standing movements. The overall risks of the area is evaluated through recognized methods of general risk assessment of National Institute of Safety and Hygiene of Spain (INSHT), methods of ergonomic evaluation, the CHECK LIST OCRA method for repetitive movements and applied OWAS awkward postures for standing, for this analysis were applied 5th and 95th percentiles, survey techniques applied to workers and interviews with staff supervision and direction, after assessing ergonomic hazards in the area aimed developing tools intended to ergonomic risk management, such as relaxation exercises, job design and plan of health monitoring, all these measures will be aimed at the reduction of absenteeism given by the appearance of diseases of musculoskeletal type.

Keywords: Ergonomics, risk of work, occupational disease, musculoskeletal injuries, cut peeled, deveined. 


\section{Plan de acción para la gestión del riesgo ergonómico en el área de pelado, corte y desvenado a los que están expuestos los trabajadores en una empacadora de camarón}

Vol. 2, núm. 1., (2018)

Luis Alberto Anchundia Santana; Robin Alejandro Torres; Gilberto Giovanni Francisco Carranza Pacheco

\section{Introducción.}

El proceso de producción del área de pelado, corte y desvenado de camarón; obliga a los trabajadores a adoptar posturas inadecuadas y exposición prolongada de pie durante toda su jornada de trabajo.

La adopción de posturas inadecuadas y exposición prolongada de pie, propias del proceso productivo, sumada la falta de una cultura de prevención o desconocimiento de los trabajadores podría generar deterioro en la salud de los colaboradores y desencadenar en enfermedades profesionales de tipo muscular y esquelético. (Muñoz Poblete, Vanegas López, \& Marchetti Pareto, 2012) (Guillén Fonseca, 2006)

La ausencia de un plan de acción para la gestión del riesgo ergonómico en el área de pelado, corte y desvenado, indudablemente llevarían al deterioro de la salud de los colaboradores dado por la no adaptación del puesto de trabajo. Por ello es de extrema importancia su desarrollo, para la gestión del riesgo ergonómico que garantice la aplicación de medidas y procedimientos a seguir en pos del cuidado de la salud de los trabajadores. (Llorca Rubio \& Gil-Monte, 2013)

El control de la exposición y los efectos generados en las actividades desarrolladas por los trabajadores de las líneas de pelado corte y desvenado, justifica la aplicación de un plan de gestión que permita la eliminación o reducción de los riesgos a los que se exponen los trabajadores en sus labores encomendadas. La aplicación de las medidas efectivas pretende la reducción de lesiones, enfermedades profesionales aumento de la eficiencia productiva, aumento 


\section{Plan de acción para la gestión del riesgo ergonómico en el área de pelado, corte y desvenado a los que están expuestos los trabajadores en una empacadora de camarón}

Vol. 2, núm. 1., (2018)

Luis Alberto Anchundia Santana; Robin Alejandro Torres; Gilberto Giovanni Francisco

Carranza Pacheco

del bienestar de los trabajadores e imagen de la empresa ante los clientes y órganos de control. (Laurig \& Vedder, 2012)

El resultado del presente proyecto de investigación se alinea al compromiso institucional y políticas internas de Empacadora, direccionadas al mejoramiento continuo y cumplimiento de la ley, así la (Asamblea Constituyente del Ecuador, 2008) señala que "La salud es un derecho que garantiza el Estado, cuya realización se vincula al ejercicio de otros derechos, entre ellos el derecho al agua, la alimentación, la educación, la cultura física, el trabajo, la seguridad social, los ambientes sanos y otros que sustentan el buen vivir" (p.16).

La aplicación de la herramienta denominada "Plan de acción para la gestión del riesgo ergonómico en el área de pelado, corte y desvenado a los que expuestos los trabajadores en una empacadora de camarón. Validación del plan, en la empacadora ", tiene como objetivo la identificación y evaluación de los factores de riesgos ergonómicos así mismo, formular propuestas de adaptación del trabajador al puesto del trabajo basado en los riesgos identificados , las directrices que se desarrollen ayudaran al mejoramiento del puesto de trabajo objeto del estudio, dando como resultado la minimización de los riesgos de tipo ergonómicos, control de enfermedades profesionales (afectaciones de tipo musculo esqueléticas) y un mejor desempeño productivo dado puestos de trabajos seguros en las líneas de pelado, corte y desvenado de Empacadora

El objetivo de la presente es identificar los riesgos ergonómicos a los que están expuestos en el área de pelado corte y desvenado en una empacadora de camarón. 


\section{Plan de acción para la gestión del riesgo ergonómico en el área de pelado, corte y desvenado a los que están expuestos los trabajadores en una empacadora de camarón}

Vol. 2, núm. 1., (2018)

Luis Alberto Anchundia Santana; Robin Alejandro Torres; Gilberto Giovanni Francisco Carranza Pacheco

\section{Metodología.}

El presente trabajo de investigación es de tipo descriptivo dado que permite seleccionar características propias del objeto de estudio.

La metodología aplicada es de tipo cuantitativo y cualitativo, estos métodos nos permiten evaluar los factores de riesgo ergonómico a los que se encuentran expuestos los trabajadores del área de pelado, corte y desvenado de Empacadora, y determinar las alteraciones posturales más frecuentes a las que se exponen los trabajadores durante su jornada laboral.

El objetivo es proponer acciones encaminadas a disminuir la incidencia de los factores de riesgos a los que están expuestos los trabajadores de esta área a fin de mejorar las condiciones de este puesto de trabajo.

En el presente proyecto utilizaremos la investigación no experimental de tipo transversal se observa el comportamiento de estas en los trabajadores del área de pelado, corte y desvenado. Esto nos permitirá plantear un plan de acción para la gestión del riesgo ergonómico a los que están expuestos los trabajadores del área de pelado, corte y desvenado de Empacadora EL objetivo será promover la salud, y el bienestar y productividad de los trabajadores de esta área.

\section{Población y muestra}

El universo considerado son 126 trabajadores de las tres líneas de pelado, corte y desvenado de Empacadora la población total de estas líneas son mujeres de recintos aledaños a la empresa 


\section{Plan de acción para la gestión del riesgo ergonómico en el área de pelado, corte y desvenado a los que están expuestos los trabajadores en una empacadora de camarón}

Vol. 2, núm. 1., (2018)

Luis Alberto Anchundia Santana; Robin Alejandro Torres; Gilberto Giovanni Francisco

Carranza Pacheco

(Jaramijo, la Sequita, y Montecristi), Dado que el universo es pequeño no se escogerá una muestra para hacer la investigación sino que se la realizará a todos los empleados de esta área.

\section{Técnica de Recolección de datos}

Las técnicas de recolección de datos aplicado al presente estudio son:

La observación directa del puesto de trabajo en las líneas de proceso en las que se desarrollan las operaciones, se elaboró encuesta directa de preguntas puntuales, en esta se incluyó a todos los trabajadores de esta área, se aplicó técnica de entrevista realizada a jefe de planta, medico ocupacional y supervisores de área, con el objetivo de poder determinar si se conoce los problemas que podrían generarse por la ausencia de gestión. Se realizó la evaluación de riesgos de esta área a través de métodos reconocidos en nuestro caso se aplicó la evaluación de riesgos a través del método general de riesgos del Instituto Nacional de Higiene del Trabajo de España (INSHT). Y a fin de tener un dato cuantitativo de la afectación real de la exposición a posturas forzadas se aplicó métodos de evaluación ergonómica reconocidos que se detallan más adelante.

Se estructuro la encuesta directa con preguntas relacionadas a nuestro objeto de estudio, se realizó preguntas a todos los trabajadores del área de Pelado corte y desvenado a fin de poder obtener información directa y necesaria para el desarrollo del presente estudio.

Se desarrolló entrevista dirigida a Jefe de Planta, Medico Ocupacional, servicio de enfermería y supervisores, se estimó conveniente recopilar datos de gestión aplicados en la actualidad en este 


\section{Plan de acción para la gestión del riesgo ergonómico en el área de pelado, corte y desvenado a los que están expuestos los trabajadores en una empacadora de camarón}

Vol. 2, núm. 1., (2018)

Luis Alberto Anchundia Santana; Robin Alejandro Torres; Gilberto Giovanni Francisco Carranza Pacheco

puesto y conocimiento por parte de los responsables y jefes de esta área de los riesgos ergonómicos a los cuales están expuestos los trabajadores.

Se aplicaron los métodos de evaluación de riesgos específicos, aplicables a la tarea a fin de poder reconocer y valorar los riesgos de exposición del puesto de trabajo se realizó la identificación de riesgos a través del método general de evaluación de riesgos laborales de España del Instituto Nacional de Higiene y Seguridad INSHT.

El objetivo principal del uso de estas herramientas es la recolección de la mayor información posible y tener una mejor visión del objeto de estudio.

Observación directa.- Gracias a la observación directa de los puestos de trabajo pudimos visualizar como los trabajadores efectúan las actividades, el ambiente laboral, la adopción de posturas, el tiempo de exposición repetitividad de la tarea y otros factores que pudieran afectar o incidir de forma negativa en el trabajo.

Métodos de evaluación ergonómica.- se evaluó la exposición cuantitativa del riesgo ergonómico a través de métodos tales como:

Método Check list ocra.- se aplicó este método para la evaluación rápida de exposición de movimientos repetitivos en miembros superiores de los obreros de esta área.

Método Owas.- este método lo aplicamos a fin de evaluar la carga postural aplicada a esta tarea. El método basa sus resultados en la observación de las diferentes posturas que los trabajadores incurren durante la tarea. 


\section{Plan de acción para la gestión del riesgo ergonómico en el área de pelado, corte y desvenado a los que están expuestos los trabajadores en una empacadora de camarón}

Vol. 2, núm. 1., (2018)

Luis Alberto Anchundia Santana; Robin Alejandro Torres; Gilberto Giovanni Francisco

Carranza Pacheco

\section{Resultados.}

Se muestran los resultados obtenidos de la encuesta realizada a los 126 trabajadores del área de pelado, corte y desvenado de Empacadora.

Se escogió una muestra de 126 trabajadores, que corresponden al 100\% de los trabajadores por considerar que es una población pequeña y con el objetivo de no tener sesgos o un margen de error mínimo, a continuación se muestran los resultados obtenidos a través de tablas estadísticas y gráficos, se indica el análisis e interpretación de resultados.

\section{Pregunta 1. ¿Cuantas horas labora durante una jornada normal de trabajo?}

El $96 \%$ de la población cumplen con un horario mayor a 8 horas, mientras un 4\% de la población labora de 6 a 8 horas siendo este grupo a la población que se encuentra en control médico o periodo de maternidad. Es decir la jornada de trabajo se extiende por un periodo mayor a 8 horas diarias.

Pregunta 2. ¿Para realizar su trabajo debe adoptar posturas forzadas de pie durante su jornada de trabajo?

De las 126 personas encuestadas correspondientes al 100\% la respuesta es que durante sus actividades laborales ellos deben permanecer de pie por lo cual se estaría incurriendo en la adopción de posturas forzadas de pie durante toda la jornada de trabajo. Se comprueba la exposición a posturas estáticas o forzadas durante la tarea. 
Plan de acción para la gestión del riesgo ergonómico en el área de pelado, corte y desvenado a los que están expuestos los trabajadores en una empacadora de camarón

Vol. 2, núm. 1., (2018)

Luis Alberto Anchundia Santana; Robin Alejandro Torres; Gilberto Giovanni Francisco Carranza Pacheco

\section{Movimientos repetitivos en la tarea de pelado corte y desvenado}

\section{Pregunta 3. ¿Cuándo realiza su trabajo aplica movimientos repetitivos para efectuar su tarea?}

De las 126 personas encuestadas la respuesta de los trabajadores fue que durante la actividad de pelado, corte y desvenado, ellos deben pelar cortar y desvenar muchas unidades (camarones), durante su jornada de trabajo por los cuales la tarea es repetitiva, basándonos en el concepto expuesto en el marco conceptual Movimientos repetitivos.

\section{Manipulación de cargas en la tarea de pelado, corte y desvenado de empacadora}

\section{Pregunta 4. ¿Realiza manipulación de cargas durante su jornada de trabajo?}

Al analizar los resultados se detecta que un $3 \%$ de los trabajadores realizan la manipulación de cargas, según observación de la tarea la operación de abastecimiento del camarón en las mesas lo realizan hombres, un $66 \%$ de la población refiere que realizan manipulación de cargas de vez en cuando y esta solo es para el abastecimiento de bandejas de agua siendo los pesos no mayores a 5 libras, el 31\% de la población expone que nunca realizan levantamiento de cargas puesto que no consideran el traslado de agua como un peso significativo y este se realiza con una periodicidad no mayor de dos a tres veces por hora.

\section{Molestias de tipo muscular}




\section{Plan de acción para la gestión del riesgo ergonómico en el área de pelado,}

corte y desvenado a los que están expuestos los trabajadores en una empacadora de camarón

Vol. 2, núm. 1., (2018)

Luis Alberto Anchundia Santana; Robin Alejandro Torres; Gilberto Giovanni Francisco

Carranza Pacheco

Pregunta 5. ¿Se ha producido como consecuencia de su trabajo molestias o dolor de tipo muscular en alguna parte de su cuerpo?

La muestra considerada en la encuesta señalan 102 personas, correspondientes al $81 \%$ de la población que en alguna ocasión han presentado molestias de tipo muscular en alguna parte del cuerpo atribuyendo estas molestias a la tarea que ejecutan.24 personas equivalente al $19 \%$ de la población no atribuyen molestias de tipo muscular a esta tarea.

Partes del cuerpo afectadas por posturas forzadas de pie y movimientos repetitivos

Pregunta 6. ¿En qué parte del cuerpo a presentado molestias que usted atribuya a su tarea?

PERSONAS QUE ACUSAN MOLESTIAS A NIVEL DEL CUELLO

El $78 \%$ de la población atribuyo molestias a nivel del cuello a la tarea. El 22\% expreso que no han tenido molestias en esta parte del cuerpo.

TRABAJADORES QUE HAN PRESENTADO MOLESTIAS A NIVEL DE LOS HOMBROS

El $67 \%$ de las personas encuestadas atribuyeron molestias a nivel de los hombros. El $33 \%$ no expresaron o atribuyeron molestias en esta zona del cuerpo.

PACIENTES QUE HAN PRESENTADO MOLESTIAS A NIVEL DE LOS BRAZOS 
Plan de acción para la gestión del riesgo ergonómico en el área de pelado, corte y desvenado a los que están expuestos los trabajadores en una empacadora de camarón

Vol. 2, núm. 1., (2018)

Luis Alberto Anchundia Santana; Robin Alejandro Torres; Gilberto Giovanni Francisco Carranza Pacheco

El $79 \%$ de los trabajadores atribuyeron molestias a nivel de los brazos al final de la jornada manifestando que en ocasiones el aumento de la producción genera este tipo de molestias en un mayor grado.

TRABAJADORES QUE HAN PRESENTADO MOLESTIAS A NIVEL DE LAS MANOS, DEDOS $Y M U \tilde{N} E C A S$

El $76 \%$ de la población atribuyo entumecimiento y dolor en alguna ocasión en esta parte del cuerpo probablemente atribuida a la repetitividad de la tarea sin embargo expresan presan que gracias a programa de pausas activas que se está implementando ha ayudado a disminuir este problema.

TRABAJADORES QUE HAN PRESENTADO MOLESTIAS A NIVEL DE LA ESPALDA

El $81 \%$ de la población expreso que en alguna ocasión han sufrido dolores atribuidos a la espalda. El $19 \%$ de los trabajadores expresaron que el dolor en otras partes del cuerpo es mayor y en esta parte esporádica.

TRABAJADORES QUE HAN PRESENTADO MOLESTIAS A NIVEL DE LA RODILLA

59\% de la población expreso molestias a nivel de la rodilla, esta condición puede ser atribuida a la posición forzada de pie, el $41 \%$ de la muestra no atribuyo mayor molestia a esta zona del cuerpo. 


\section{Plan de acción para la gestión del riesgo ergonómico en el área de pelado,}

corte y desvenado a los que están expuestos los trabajadores en una empacadora de camarón

Vol. 2, núm. 1., (2018)

Luis Alberto Anchundia Santana; Robin Alejandro Torres; Gilberto Giovanni Francisco

Carranza Pacheco

TRABAJADORES QUE HAN PRESENTADO MOLESTIAS A NIVEL DE PIERNAS, PIE O

TOBILLOS

El $90 \%$ de la muestra expreso que en varias ocasiones dada la exposición terminan el turno de trabajo con dolor en esta parte del cuerpo, atribuyen este problema a la posición forzada de pie.

PORCENTAJE DE LAS MOLESTIAS MUSCULARES QUE SE HAN PRESENTADO EN LAS TAREAS DE PELADO CORTE Y DESVENADO

Se consideró para la encuesta las partes del cuerpo con posible afectación en la tarea, en base a los resultados obtenidos podemos decir que el área de mayor afectación de la tarea han sido las piernas pies y tobillos con un $90 \%$. Le siguen los dolores de espalda con un $81 \%$ y una afectación del 79\% de la población atribuyen molestias a nivel del cuello, el $78 \%$ atribuye molestias a nivel de los brazos un $76 \%$ manos, un $66 \%$ atribuyen molestias en los hombros y un $58 \%$ rodillas.

\section{Nivel de ausentismo del puesto de trabajo}

Pregunta 7. ¿Ha tenido que ausentarse de su puesto de trabajo debido a problemas de tipo Muscular?

El $60 \%$, alegan que en alguna ocasión han tenido que ausentarse debido a molestias que ellos atribuyen a la tarea, sin embargo, esta no ha sido tan representativa o notorio dado que este ausentismo no se ha presentado de manera masiva y el número de personas es de 126 , por lo que 
Plan de acción para la gestión del riesgo ergonómico en el área de pelado, corte y desvenado a los que están expuestos los trabajadores en una empacadora de camarón

Vol. 2, núm. 1., (2018)

Luis Alberto Anchundia Santana; Robin Alejandro Torres; Gilberto Giovanni Francisco Carranza Pacheco

no ha afectado en forma representativa la producción, además no se ha llevado seguimiento a esta condición generada.

\section{Capacitación desarrollada en riesgos de la tarea}

Pregunta 8. ¿Ha recibido algún tipo de información y capacitación referente a los riesgos existentes en su puesto de trabajo?

El $100 \%$ de la población ha recibido capacitación de los riesgos de la tareas, la empresa está implementando programas de seguridad en caminados a la prevención de riesgos y mejoramiento del ambiente de trabajo.

\section{Personal que Conoce los riesgos de la tarea}

Pregunta 9. ¿Conoce los riesgos o efectos generados por las tareas que ejecuta en su puesto de trabajo?

De acuerdo a lo señalado en la figura 20 el $78 \%$ de la población encuestada, alega conocer los riesgos de la tarea, sin embargo al parecer no se ha hecho énfasis en las consecuencias de estos en su salud, $22 \%$ de la población dice conocer algo los riesgos que los afectan en el puesto de trabajo, es necesario medir y reforzar la efectividad de la capacitación por parte del área de RRHH.

Implementación de medidas de prevención 


\section{Plan de acción para la gestión del riesgo ergonómico en el área de pelado, corte y desvenado a los que están expuestos los trabajadores en una empacadora de camarón}

Vol. 2, núm. 1., (2018)

Luis Alberto Anchundia Santana; Robin Alejandro Torres; Gilberto Giovanni Francisco

Carranza Pacheco

Pregunta 10. ¿Considera necesario la aplicación de medidas de protección frente al riesgo ergonómico en su puesto de trabajo?

El $100 \%$ de la población indico, su total acuerdo a la necesidad de implementación de un plan de prevención del riesgo ergonómico, a fin de poder evitar las afectaciones dadas en el puesto de trabajo.

\section{Conclusiones.}

De acuerdo a los resultados obtenidos, en el presente estudio realizado en el área de pelado corte y desvenado, se pudo demostrar la afectación que se está generando en los trabajadores debido a la exposición a los factores de riesgo de tipo ergonómico.

El factor de riesgo o exposición a posturas forzadas y movimientos repetitivos en esta área es inaceptable por lo cual deben aplicarse medidas de manera inmediata. Aun existiendo una unidad de seguridad en la empresa, el direccionamiento a la gestión de factores de riesgos ergonómicos debe ser mejorada, dado que se pudo evidenciar que las acciones aplicadas a la empresa no han sido en un $100 \%$ efectivas

Que el déficit de gestión o aplicación de medidas preventivas en el área de pelado corte y desvenado, puede ser sujeto de sanciones a través del Instituto Ecuatoriano de Seguridad Social por la exposición de los trabajadores a factores de riesgos intolerables. Las mesas de trabajo no están diseñadas acorde a las condiciones antropométricas de las trabajadoras de estatura alta, esta 


\section{Plan de acción para la gestión del riesgo ergonómico en el área de pelado, corte y desvenado a los que están expuestos los trabajadores en una empacadora de camarón}

Vol. 2, núm. 1., (2018)

Luis Alberto Anchundia Santana; Robin Alejandro Torres; Gilberto Giovanni Francisco Carranza Pacheco

condición obliga a estas trabajadoras a adoptar posturas incorrectas o no adecuadas, se encuentran adecuadas para trabajadoras de talla baja.

Los riesgos de exposición por condiciones de trabajo tales como pisos húmedos o mojados, manejo de herramientas cortantes, o golpes por manejo de coches en el área deben ser de igual forma gestionada su corrección o minimización del riesgo.

\section{Bibliografía.}

Asamblea Constituyente del Ecuador. (2008). Constitucion del Ecuador. Montecristi: autor.

Guillén Fonseca, M. (2006). Ergonomía y la relación con los factores de riesgo en salud ocupacional. Revista Cubana de Enfermería, 22(4), 15-17.

Laurig, W., \& Vedder, J. (2012). ERGONOMIA. Madrid: Instituto Nacional de Seguridad e Higiene en el Trabajo (INSHT).

Llorca Rubio, J., \& Gil-Monte, P. (2013). Prevención de riesgos laborales y su relación con el género de los trabajadores. Saúde Soc. São Paulo, 22(3), 727-735.

Muñoz Poblete, C., Vanegas López, J., \& Marchetti Pareto, N. (2012). Factores de riesgo ergonómico y su relación con dolor musculoesquelético de columna vertebral: basado en la primera encuesta nacional de condiciones de empleo, equidad, trabajo, salud y calidad de vida de los trabajadores y trabajadoras en Chile (ENETS) 2. Medicina y Seguridad del Trabajo, 58(228), 194-204. 